\title{
Optimal sensor placement for active control of floor vibration considering spillover effect associated with modal filtering
}

$\operatorname{AUTHOR}(S):$

Kai, Xue; Igarashi, Akira; Kachi, Takahiro

\section{CITATION:}

Kai, Xue ...[et al]. Optimal sensor placement for active control of floor vibration

considering spillover effect associated with modal filtering. Engineering Structures 2018,

165: 198-209

ISSUE DATE:

2018-06-15

URL:

http://hdl.handle.net/2433/230326

\section{RIGHT:}

(c) 2018. This manuscript version is made available under the CC-BY-NC-ND 4.0 license

http://creativecommons.org/licenses/by-nc-nd/4.0/; The full-text file will be made open to the public on 15 June 2020 in accordance with publisher's 'Terms and Conditions for Self-Archiving'.; この論文は出版社版でありません。引用の際 には出版社版をご確認ご利用ください。; This is not the published version. Please cite only the published version. 


\title{
Optimal sensor placement for active control of floor vibration considering spillover effect associated with modal filtering
}

\author{
Kai Xue ${ }^{1}$, Akira Igarashi ${ }^{2}$ and Takahiro Kachi ${ }^{3}$ \\ ${ }^{1}$ Department of Urban Management, Kyoto University, Kyoto, Japan \\ ${ }^{2}$ Disaster Prevention Research Institute, Kyoto University, Kyoto, Japan \\ ${ }^{3}$ Sumitomo Riko Co. Ltd., Aichi, Japan \\ xue.kai.57r@st.kyoto-u.ac.jp, igarashi.akira.7m@kyoto-u.ac.jp, \\ takahiro.kachi@jp.sumitomoriko.com
}

\section{ABSTRACT}

Application of the modal filtering technique to active control can be effectively used to suppress vibration of floor panels by increasing the damping of the natural vibration modes involved. Limitation of this method is related to the spillover effect caused by the neglected modes. In this study, a sensor placement determination procedure is developed so that enhancement of the separation performance of the modeled modes and reduction of the spillover effect of the truncated modes in modal filtering are achieved at the same time by combining the minimum spillover method, effective independence method (EFI) and modal assurance criterion (MAC). Performance of the proposed procedure is investigated by conducting verification tests to control vibration of a rectangular steel plate with the modal filtering technique, and the sensor placement determined by the proposed approach achieves preferable results than other two sensor placement cases. The effectiveness of the proposed technique is also verified by state feedback control for individual modes. Using the proposed approach, effective active control of the steel plate vibration is successfully achieved.

\section{Keywords: Sensor Placement, Plate Vibration Suppression, Modal Filtering, Spillover Effect, active control.}

\section{Introduction}

The floor impact sound problem in residential buildings is essentially a serviceability problem in terms of annoying the users and rarely affect the fatigue damage or safety of structures since the main cause of the floor impact sound is the vibration of floor members induced by external actions [1,2]. Application of dynamic response control techniques to the floor members can be effective in suppressing the sound. Application of the active control techniques using sensors, actuators and a controller can be a promising approach because of its high effectiveness in vibration cancellation and the ability to deal with multiple modal responses in a simple manner compared to the passive and semi-active control methods [3, 4].

Research on utilizing active vibration control (AVC) technology towards human-induced vibration of floors can be seen in some recent trials $[1,7,8,9,10,11]$. Depending on the frequency of the first mode, floor can be divided into 'low frequency floors' and 'high frequency floors', and most of the research until now focus on 'low frequency floors' because the progress of light weight and long span construction which inherently have less damping [5]. Direct-Output Feedback controllers which multiply the sensor outputs by a gain matrix to produce the actuator commands are usually chosen for the floor vibration control [6]. One of the earliest applications of AVC is presented by Hanagan using the direct velocity feedback (DVF) controller to reduce the floor motion, achieving vibration reduction of over $75 \%[3,4]$. Since then, further work has improved on this controller. For example, Díaz and Reynolds presented a compensated acceleration feedback control algorithm implemented on an office floor in which a phase-lag compensator is applied to the acceleration outputs such that the properties of high damping at the fundamental vibration mode and high stability margins are achieved, the amount of time spent above a response factor (a common vibration limit for high quality office environments) was reduced by $97 \%$ for the in-service testing [7]. Díaz and Reynolds also investigated the direct velocity feedback with feed-through term controller in order to make the system more robust with respect to stability and achieve excellent reduction in vibration response [8]. Donald Nyawako introduced a disturbance observer that is used to suppress the off-resonance vibration combined with the DVF controller and the results showed that the inclusion of the disturbance observer with an outer loop DVF has improved the vibration mitigation performance by about $3.5 \%$ at resonance and $6-10 \%$ off-resonance [9].

However, the excessive level of vibration is usually a problem of a wide area not just a single location, which requires using of several collocated actuator-sensor pairs to achieve the vibration reduction requirement. Multiple single-input and single-output (SISO) control schemes which are designed for each location independently was first investigated, however, since the structural system does not act independently at each control location, it was shown to have limited efficiency in which a reduction of the control gain of each SISO is needed to guarantee stability [10]. Multi-input and multi-output (MIMO) was then used for the global vibration control of floor motion. These DVF MIMO control strategy find the optimal gain matrix and the optimal location 
for a predefined number of actuators and sensors obtained by minimizing a performance index PI. It was observed that MIMO control improved the results compared with the SISO control strategy [11].

On the other hand, 'high frequency floors' and Model Based (MB) controller have not been deeply studied for the humaninduced vibrations of floor. Although the building floor can be theoretically regarded as a distributed-parameter system, it is possible to represent its dynamics by a discrete system that allows the application of the technique referred to as the modal filtering (MF). The concept of MF is that the coupled equations of motion of a discrete system are decoupled into a set of independent modes in the modal space $[12,13,14]$. Using this technique, the modal control forces can be independently designed for each mode through feed-back control and applied by actuators. Since it is not practical and not necessary to model all the modes, a few number of lower modes or modes in the bandwidth of interest are considered to be controlled by this method. However, the neglected modes are still present in the system and the truncation of the modes may cause a spillover effect when the system is subjected to disturbance excitation or control force [15]. The spillover effect should be avoided since it may cause system instability and poor control performance [16, 17].

For successful application of MF technique for floor system vibration control, the placement of sensors and actuators to reduce the associated noise and spillover effect and to enhance the performance of the system as well as its fatigue durability is of great significance [18]. While the number of sensors should be greater or equal to the total number of the modes considered in modal filtering, use of a large number of sensors is not recommended for the economic and aesthetic considerations. For the case of dynamic response control of a system with classical damping, it is possible to point out the following four issues that should be considered in determining the sensor placements. The first one is the observability, which implies that the sensors should be located in the vicinity of the anti-nodes of the natural modes to be measured. The second one is the state estimate ability [19], which implies that the covariance matrix of the estimate errors should be as small as possible with the effect of white noise in the measurement signal by the sensors. The third one is the orthogonality, which is considered to ensure the principle of modal filtering to rigorously hold and enhance robustness against the error of modal shape estimation. The last one is the spillover, as previously mentioned. The spillover observed by the sensors should be as small as possible to avoid instability caused by the truncated modes.

The objective of the study described in this paper is to develop a sensor placement procedure to deal with these four problems. The minimum spillover method, the effective independence method and the modal assurance criterion used as background techniques are introduced in Sec. 2. The proposed sensor placement method that enhances the separation performance of the modeled modes and reduces the spillover effect of the neglected modes is developed in Sec. 3. A brief introduction of phase compensated direct velocity feedback control is shown in Sec. 4. Some results of experimental tests are discussed in Sec. 5.

\section{Preliminaries}

\subsection{The minimum spillover method}

The MF technique is based on the idea of coordinate transformation, where the signal measured by the sensors can be expressed as a linear combination of sinusoidal responses in multiple natural vibration modes. This situation is expressed as follows:

$$
\mathbf{u}(\mathrm{t})=\sum_{n=1}^{N} A_{n} \boldsymbol{\Psi}_{n} \cos \left(\omega_{n} t-\gamma_{n}\right)=\sum_{n=1}^{N} q_{n}(t) \boldsymbol{\Psi}_{n}
$$

where $\mathbf{u}(\mathrm{t})$ is the response measured by sensors represented as a vector, $N$ is the number of natural modes to be considered, $A_{n}$ is the real amplitude, $\boldsymbol{\Psi}_{n}$ is the modal shape vector, $\gamma_{n}$ is the phase, $\omega_{n}$ is the natural angular frequency and $n$ denotes that the variable is for the $n$th mode. The symbol $q_{n}(t)$ represents the generalized coordinate of the $n$th mode that represents the $n$th mode response $A_{n} \cos \left(\omega_{n} t-\gamma_{n}\right)$ in this case.

The number of the modes $N$ is assumed to be a finite integer. Let us assume that the number of the controlled modes be $M$ such that $M<N$. In this case, the response $\mathbf{u}(\mathrm{t})$ can be divided into a controlled part and a residual part [20, 21], which can be expressed in a matrix-form:

$$
\{\mathrm{u}\}=\left[\Psi_{m}\right]\left\{q_{m}\right\}+\left[\Psi_{u m}\right]\left\{q_{u m}\right\}
$$

where $\{\mathrm{u}\}$ is an $S$-dimensional vector, $S$ is the number of sensors such that $S \geq M$, the size of the matrix $\left[\Psi_{m}\right]$ is $S \times M$, that of $\left[\Psi_{u m}\right]$ is $S \times(N-M),\left\{q_{m}\right\}$ is an $M$-dimensional vector and $\left\{q_{u m}\right\}$ is an $(N-M)$-dimensional vector.

From Eqn. (2):

$$
\left\{q_{m}\right\}=\left(\left[\Psi_{m}\right]^{T}\left[\Psi_{m}\right]\right)^{-1}\left[\Psi_{m}\right]^{T}\{u\}-\left(\left[\Psi_{m}\right]^{T}\left[\Psi_{m}\right]\right)^{-1}\left[\Psi_{m}\right]^{T}\left[\Psi_{u m}\right]\left\{q_{u m}\right\}
$$

Note that $\left(\left[\Psi_{m}\right]^{T}\left[\Psi_{m}\right]\right)^{-1}\left[\Psi_{m}\right]^{T}$ is the generalized inverse of the modal matrix $\left[\Psi_{m}\right][22]$. The second term on the right 
hand side of Eqn. (3) is referred to as the residual part. If the spillover effects are sufficiently small and negligible, Eqn. (3) can be expressed as:

$$
\left\{q_{m}\right\}=\left(\left[\Psi_{m}\right]^{T}\left[\Psi_{m}\right]\right)^{-1}\left[\Psi_{m}\right]^{T}\{u\}
$$

In order for $\left\{q_{m}\right\}$ to be acceptably expressed by Eqn. (4), the modal shape matrix $\left[\Psi_{u m}\right]$ included in the residual part should be altered by adjusting the sensor placement. The spillover term $\{\Delta\}$ is defined as the residual part with the negative sign:

$$
\{\Delta\}=\left(\left[\Psi_{m}\right]^{T}\left[\Psi_{m}\right]\right)^{-1}\left[\Psi_{m}\right]^{T}\left[\Psi_{u m}\right]\left\{q_{u m}\right\}=\left[\Psi_{\text {spill }}\right]\left\{q_{u m}\right\}
$$

Minimization of the spillover can be achieved by using the norm of the spillover term $\{\Delta\}$ as the objective function:

$$
\|\Delta\|^{2}=\left\{q_{u m}\right\}^{T}\left[\Psi_{\text {spill }}\right]^{T}\left[\Psi_{\text {spill }}\right]\left\{q_{u m}\right\}=\left\{q_{u m}\right\}^{T}\left[\Psi_{\text {spi }}\right]\left\{q_{u m}\right\}
$$

For any $\left\{q_{u m}\right\}$, the following inequality holds:

$$
\lambda_{\min }\left(\left[\Psi_{s p i}\right]\right) \leqslant \frac{\left\{q_{u m}\right\}^{T}\left[\Psi_{s p i}\right]\left\{q_{u m}\right\}}{\left\{q_{u m}\right\}^{T}\left\{q_{u m}\right\}} \leqslant \lambda_{\max }\left(\left[\Psi_{s p i}\right]\right)
$$

where $\lambda_{\min }\left(\left[\Psi_{s p i}\right]\right)$ and $\lambda_{\max }\left(\left[\Psi_{s p i}\right]\right)$ are the minimum and maximum eigenvalues of $\left[\Psi_{s p i}\right]$, respectively. In order to minimize the spillover effect, sensors must be arranged to achieve the minimum value of $\lambda_{\max }\left(\left[\Psi_{s p i}\right]\right)$, which means that the sensor placement is to be determined using the following objective function [20,23]:

$$
J_{s p i}=\lambda_{\max }\left(\left[\Psi_{\text {spi }}\right]\right) \rightarrow \text { minimize }
$$

Note that the presence of noise in the measurement signals is not assumed in the formulation. Orthogonality of the modal matrix $\left[\Psi_{m}\right]$ is not assumed in this approach either. Also, since an enormous number of possible sensor locations have to be considered in the algorithm in practice, computational efficiency is required in the determination procedure of the optimal sensor location to achieve minimum of the objective function $J_{s p i}$.

The minimum spillover method can also be developed for searching the optimal actuator placement if more than one actuators are used. If the number of actuators is assumed to be $N_{a c}$, the modal control forces of the control modes $\left\{f_{m c}\right\}$ and the residual modes $\left\{f_{r}\right\}$ can be expressed as:

$$
\left\{f_{m c}\right\}=\left[B_{c}\right]\{V\}
$$

And:

$$
\left\{f_{r}\right\}=\left[B_{r}\right]\{V\}
$$

where $\left\{f_{m c}\right\}$ and $\left\{f_{r}\right\}$ are $M$-dimensional and $(N-M)$-dimensional vectors, respectively. $\{V\}$ is the control force generated by the actuators represented as an $N_{a c}$-dimensional vector. The size of the mode shape matrix $\left[B_{c}\right]$ is $N_{a c} \times M$, and that of $\left[B_{r}\right]$ is $N_{a c} \times(N-M)$.

Combining Eqn. (9) with Eqn. (10) gives:

$$
\left\{f_{r}\right\}=\left[B_{r}\right]\left(\left[B_{c}\right]^{T}\left[B_{c}\right]\right)^{-1}\left[B_{c}\right]^{T}\left\{f_{m c}\right\}
$$

A small control spillover effect can be achieved by altering the position of actuators. As discussed in the observation spillover, minimization of the control spillover can be achieved by using the norm of the spillover term $\left\{\Delta_{c}\right\}$ as the objective function: 
in which $\left[B_{\text {spili }}\right]=\left[B_{r}\right]\left(\left[B_{c}\right]^{T}\left[B_{c}\right]\right)^{-1}\left[B_{c}\right]^{T}$. For any $\left\{f_{m c}\right\}$, the following inequality holds:

$$
\lambda_{\min }\left(\left[B_{s p i}\right]\right) \leq \frac{\left\{f_{m c}\right\}^{T}\left[B_{s p i}\right]\left\{f_{m c}\right\}}{\left\{f_{m c}\right\}^{T}\left\{f_{m c}\right\}} \leq \lambda_{\max }\left(\left[B_{s p i}\right]\right)
$$

where $\lambda_{\min }\left(\left[B_{s p i}\right]\right)$ and $\lambda_{\max }\left(\left[B_{s p i}\right]\right)$ are the minimum and maximum eigenvalue $\left\{B_{s} \oint_{i}\right]$, respectively. The actuator placement is determined using the following objective function:

$$
J_{\text {spiac }}=\lambda_{\max }\left(\left[B_{\text {spi }}\right]\right) \rightarrow \text { minimize }
$$

\subsection{The effective independence method (EFI)}

In the effective independence method (EFI), the candidate sensor locations are ranked according to their contribution to the linear independence of the target modal partitions in an iterative manner [24,25]. The trace and determinant of the related Fisher information matrix are maximized by this method, leading to improved estimates of the concerned modes in the presence of white noise. The candidate solution is narrowed by removing the sensor locations that contribute the least of all the position sensor locations to the linear independence of the target modal partitions [19, 26,27]. The output of the sensors with additive white noise can be written as:

$$
\{\mathrm{u}\}=\left[\Psi_{N}\right]\left\{q_{N}\right\}+\{\varepsilon\}=\mathbf{H}\left(q_{N}\right)+\{\varepsilon\}
$$

where the symbol $\mathbf{H}$ represents the process measurement and $\{\varepsilon\}$ is a stationary Gaussian white noise with zero mean and a variance of $\left[\psi_{0}^{2}\right]$. In EFI analysis, the covariance matrix of the estimate error is computed as follows:

$$
\mathrm{E}\left[\left(\boldsymbol{q}_{N}-\hat{\boldsymbol{q}}_{N}\right)\left(\boldsymbol{q}_{N}-\hat{\boldsymbol{q}}_{N}\right)^{T}\right]=\left[\left(\frac{\partial \mathbf{H}}{\partial \boldsymbol{q}_{N}}\right)\left[\psi_{0}^{2}\right]^{-1}\left(\frac{\partial \mathbf{H}}{\partial \boldsymbol{q}_{N}}\right)\right]^{-1}=\left[\frac{1}{\psi_{0}^{2}}\left[\Psi_{N}\right]^{T}\left[\Psi_{N}\right]\right]^{-1}=\mathrm{Q}^{-1}
$$

in which $\hat{q}_{N}$ is the unbiased estimator of $q_{N}$, and $\mathrm{Q}$ is the Fisher information matrix. Maximizing $\mathrm{Q}$ is assumed to lead to the best state estimate of $q_{N}$. The first step of the procedure is to find the solution of the eigenvalue equation:

$$
\left[\left[\Psi_{N}\right]^{T}\left[\Psi_{N}\right]-\lambda \mathbf{I}\right] \boldsymbol{\Psi}=0
$$

where $\lambda$ is the diagonal eigenvalue matrix, $\boldsymbol{\Psi}$ is the matrix consisting of the orthogonal eigenvectors such that $\boldsymbol{\Psi}^{\boldsymbol{T}} \boldsymbol{\Psi}=\mathbf{I}$. The EFI coefficients of the candidate sensor locations are given by:

$$
E_{D}=\left(\left[\Psi_{N}\right] \boldsymbol{\Psi}\right) \odot\left(\left[\Psi_{N}\right] \boldsymbol{\Psi}\right) \boldsymbol{\lambda}^{-1} * \mathbf{1}
$$

where the symbol $\odot$ represents a term-by-term matrix multiplication. $\mathbf{1}$ is an $n$-dimensional column vector with all the elements of unity. $E_{D}$ is referred to as the effective independence distribution of the candidate sensor set. The $i$ th term within $E_{D}$ is the fractional contribution of the $i$ th sensor location to the linear independence of the modal matrix $\left[\Psi_{N}\right]$. The indices $E_{D}$ can also be calculated by:

$$
E_{D}=\left[\Psi_{N}\right]\left(\left[\Psi_{N}\right]^{T}\left[\Psi_{N}\right]\right)^{-1}\left[\Psi_{N}\right]^{T}
$$

Then the procedure is employed to sort the elements of the $E_{D}$ vector and to remove the smallest component corresponding to a candidate sensor location. The vector $E_{D}$ is updated based on the new modal shape matrix, and the process is repeated iteratively until the number of remaining sensor locations reaches to a pre-defined value.

Note that the purpose of EFI is to maximize information on the designated modes through the optimal configuration of 
sensors, while the spillover caused by the truncated modes is not considered.

\subsection{Modal assurance criterion (MAC)}

The MAC is defined as a scalar value representing the degree of consistency (linearity) between modal shape vectors [28]. For two modal shape vectors $\Psi_{\mathrm{a}}$ and $\Psi_{\mathrm{b}}$, the MAC value is defined by:

$$
\operatorname{MAC}\left(\Psi_{a}, \Psi_{b}\right)=\frac{\left|\Psi_{a}{ }^{H} \Psi_{b}\right|^{2}}{\left\|\Psi_{a}\right\|^{2}\left\|\Psi_{b}\right\|^{2}}
$$

The modal assurance criterion takes on values in the range between zero - representing mutual orthogonality of modal shape vectors, and unity - representing linear dependence of the two vectors. Therefore, the sensor placement with the greatest degree of independence can be found by solving the optimization problem for the following objective function:

$$
J_{\text {mac }}=\sum_{a=1}^{M-1} \sum_{b=a+1}^{M} \operatorname{MAC}\left(\Psi_{a}, \Psi_{b}\right) \rightarrow \text { minimize }
$$

where $M$ is the total modes to be considered. The sensor placement for which the sum of $\operatorname{MAC}\left(\Psi_{a}, \Psi_{b}\right)$ for all different $a$ and $b$ achieves the minimum is supposed to be closest to the one that satisfies the mutual orthogonality condition.

\section{Procedure of determination of optimal sensor placement}

\subsection{Problem statement}

In the case of floor vibration, possible sensor location candidates are assumed to be the hypothetical rectangular grid points on the floor surface with a sufficiently fine mesh size. The values of the modal shape of the plate representing the floor are treated as vector components corresponding to the grid points, and the plate is approximated as a multi-degree-of freedom (MDOF) system. Therefore, the number of sensor placement candidates is equal to the number of the grid points of the plate, denoted by $L$. Since the value of $L$ is very large in practice, the number of modes to be considered $N$, is assumed to be divided into two parts-the control modes and the residual modes, as in Eqn. (2). If the number of control modes is assumed to be $M, N-M$ is the number of residual modes. For the purpose of filtering out all the control modes, usually $S \geq M$ should be satisfied, where $S$ is the number of sensors. As stated in Sec. 1, state estimate ability, orthogonality, observability as well as spillover should be considered in determining the sensor placement for the use of modal filtering.

\subsection{Sensor placement}

As the first step, EFI is used for the state estimate ability as well as decreasing the number of sensor placement candidates. The number of sensor candidates is then reduced from a large set $L$ to a pre-defined number $P$ by removing those candidates which contribute least to the state estimate ability of the control modes in an iterative manner. The value of $P$ is specified to be sufficiently small for the use of the minimum spillover method.

Then for the $P$ candidate positions and $S$ sensors, all the possible sensor placements are ranked by the minimum spillover method in the order of the severity of spillover, implying the value of $A_{s p i}=\lambda_{\max }\left(\left[\Psi_{s p i}\right]\right)$ in Eqn. (7) in an increasing manner. 


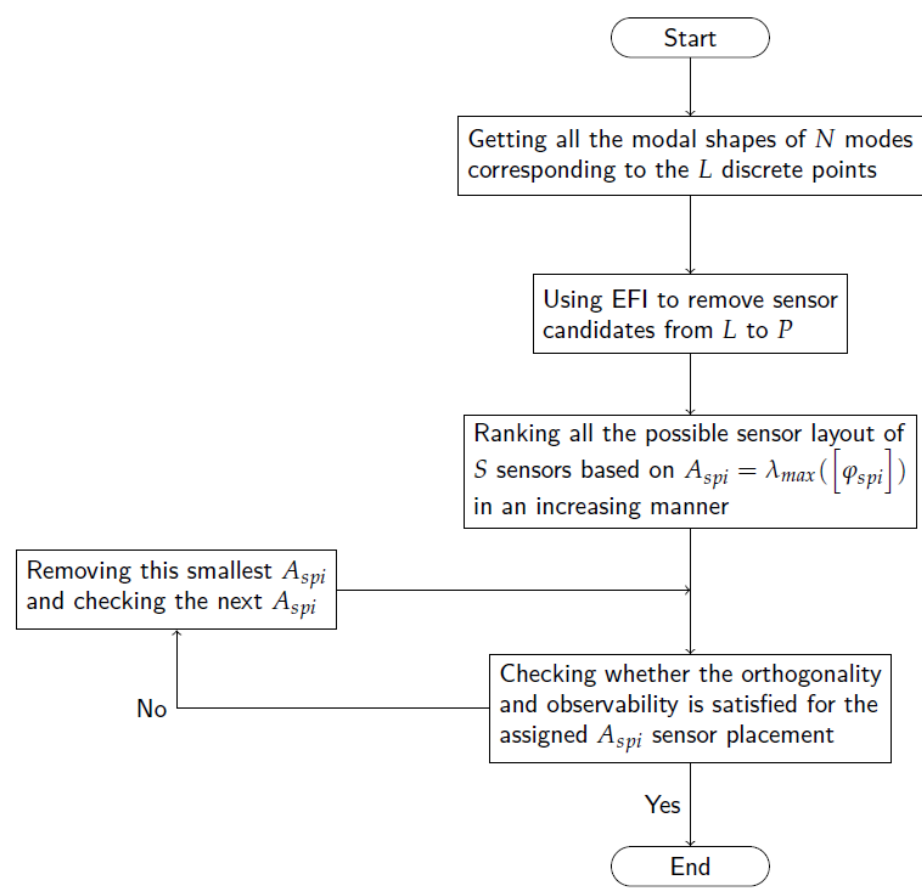

Fig. 1. Flow chart of the sensor placement design.

In the final step, observability and orthogonality are checked for the smallest $\lambda_{\max }\left(\left[\Psi_{s p i}\right]\right)$ sensor placement, and the index $A_{\text {mac }}$ is defined by $A_{\text {mac }}=\sum_{a=1}^{M-1} \Sigma_{b=a+1}^{M} \operatorname{MAC}\left(\Psi_{a}, \Psi_{b}\right)$ here. If $A_{\text {mac }}$ is sufficiently small and the system is observable, the sensor placement is the optimal one. Otherwise, if $A_{m a c}$ is greater than the tolerance value such as 0.1 , this sensor placement is discarded and the next smallest sensor placement is examined and calculated by the same procedure until an acceptable value of $A_{m a c}$ is obtained. If $A_{m c a}$ is sufficiently small, it is like to that the observability is also satisfied. The flow chart of the proposed design for choosing the optimal sensor placement is shown in Fig. 1.

\subsection{Actuator placement}

In order to achieve improved control performance, the EFI method is firstly used to reduce the actuator placement candidate from the total number of the grid points $L$ to a pre-defined number $P_{a c t}$, this removes the candidates which contribute least to excite the control modes such that controllability can be guaranteed.

Then for the $P$ candidate locations and $N_{a c}$ actuators, the minimum spillover method is utilized for all the possible actuator placement and the one with the minimum $J_{\text {spiac }}$ of Eqn. (14) is treated as the optimal actuator placement.

\section{Basic control background}

\subsection{MDVF control strategy}

If the dynamics of the actuator are not considered in the real system, the MDVF (Modal Direct Velocity Feedback) control strategy can be a natural choice due to its readily established simple implementation. The MDVF control indicates that the control force for the $j$ th mode $f_{j}(t)(j=1,2 \ldots)$ is defined to be proportional to the velocity of the $j$ th response $\dot{q}_{j}(t)$ obtained by utilizing the modal filtering to the dynamic response $\mathbf{u}(t)$ measured by the sensors, as:

$$
f_{j}(t)=-\beta_{j} \dot{q}_{j}(t)
$$

where the positive value $\beta_{j}$ is the gain for the $j$ th mode. The damping ratio of $j$ th mode can be increased by this procedure. Therefore, the control force for multiple modes, denoted by $f(t)$, is calculated by: 


$$
f(t)=f_{1}(t)+f_{2}(t)+f_{3}(t)+\cdots=\left(-\beta_{1} \dot{q}_{1}(t)\right)+\left(-\beta_{2} \dot{q}_{2}(t)\right)+\left(-\beta_{3} \dot{q}_{3}(t)\right)+\cdots
$$

The response reduction effect for each mode is achieved by choosing optimal sufficiently large value of the control gain $\beta_{j}$.

\subsection{Phase compensated MDVF control strategy}

It is assumed that the filtered mode can be approximately seemed as a single-degree-of-freedom system after modal filtering, as:

$$
m_{j} \ddot{q}_{j}(t)+c_{j} \dot{q}_{j}(t)+k_{j} q_{j}(t)=f_{c j}(t)+f_{d j}(t)
$$

where $m_{j}, c_{j}$ and $k_{j}$ are the modal mass, modal damping coefficient and modal stiffness of the jth mode, respectively, $f_{c j}(t)$ is the modal control force and $f_{d j}(t)$ is the modal disturbance. The modal coordinate $q_{j}(t)$ is in fact a damped sinusoidal response, which makes it easier to integrate the filtered response from acceleration to velocity. Note that the dynamics of actuator or other dynamics components involved in the whole system cannot be ignored during the real time control, therefore the amplitude and phase of the response $\dot{q}_{j}(t)$ for each mode would be changed, and in this case, the second order linear differential equation can be written as:

$$
m_{j} \ddot{q}_{j}(t)+c_{j} \dot{q}_{j}(t)+k_{j} q_{j}(t)=-\beta_{j} \alpha_{j} \dot{q}_{j}\left(t-t_{0 j}\right)+f_{d j}(t)
$$

where $\alpha_{j}$ and $t_{0 j}$ are the scaler of amplitude changed and phase lag of $j$ th mode, respectively. By transforming both side of Eqn. (26) into its Laplace domain, it can be expressed as:

$$
s^{2} m_{j} q_{j}(s)+s\left(c_{j}+e^{-s t_{0 j}} \beta_{j} \alpha_{j}\right) q_{j}(s)+k_{j} q_{j}(s)=f_{d j}(s)
$$

This means that the damping ratio of the target mode will be increased when $t_{0 j}=0$. However, if $t_{0 j} \neq 0$, the poles of the mode may lie in the right half-plane, implying instability. So a modified MDVF controller designed to increase the closed-loop system stability and to make the system more amenable to the introduction of significant damping for each mode to be controlled through phase compensation is needed. In this paper, a proportional-integral (PI) technique is used by applying a weighted linear combination of integration of the target modal control velocity with itself. The procedure of the PI phase compensation for the $j$ th mode is shown in Fig. 2. For simplicity, only the actuator is shown as the dynamics component.

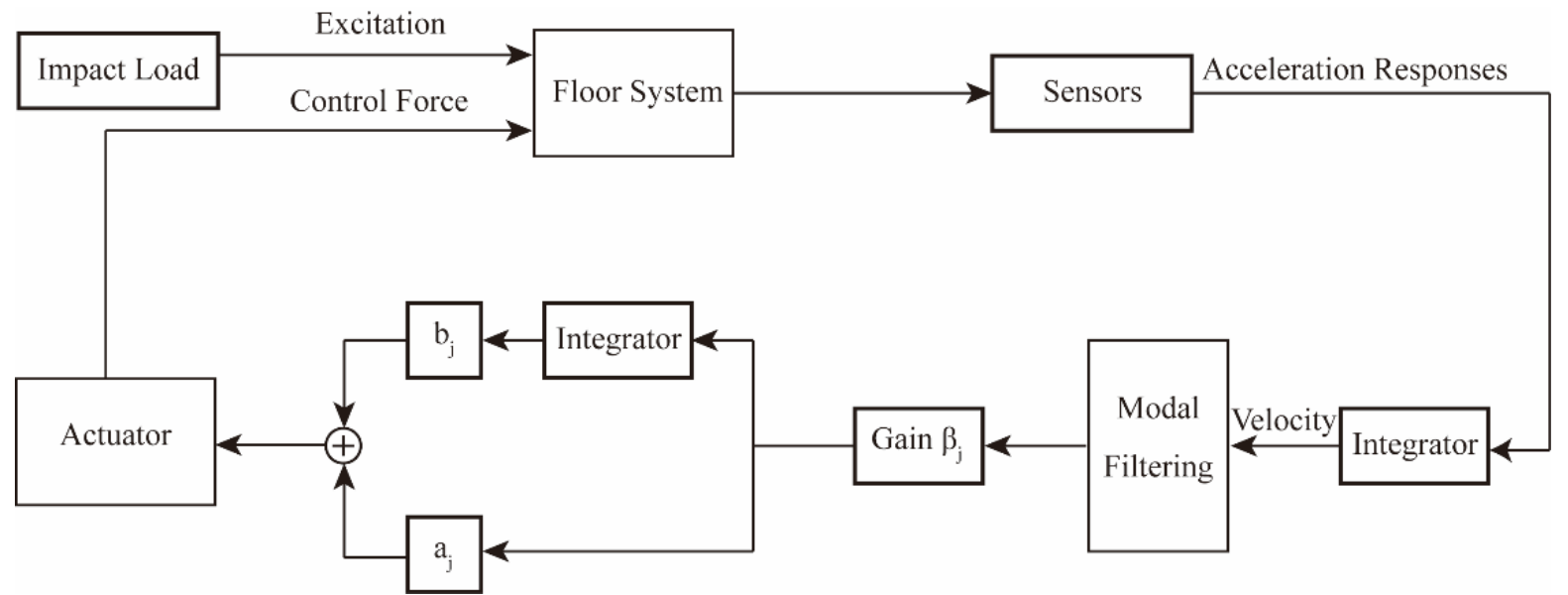

Fig. 2. Phase compensated active control of $j$ th mode.

\section{Verification test}




\subsection{Test setup and experimental identification}

The sensor placement approach was applied to a steel plate specimen with all four edges clamped. The system is shown in

Fig. 3. The system consists of a $840 \mathrm{~mm} \times 840 \mathrm{~mm}$ steel plate with a thickness of $4.5 \mathrm{~mm}$. Dimensions of the steel plate specimen are shown in Fig. 4. A square $9 \times 9$ grid mesh was defined to specify $L=81$ sensor location candidates on the surface of the steel plate, as shown in Fig. 5.

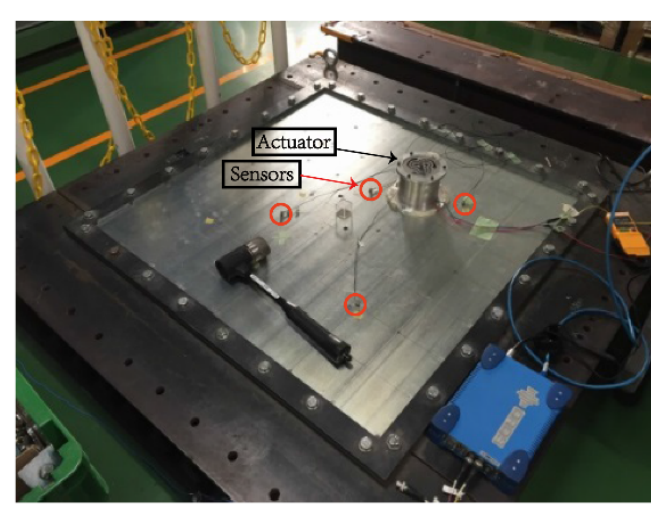

Fig. 3. Experimental set-up.

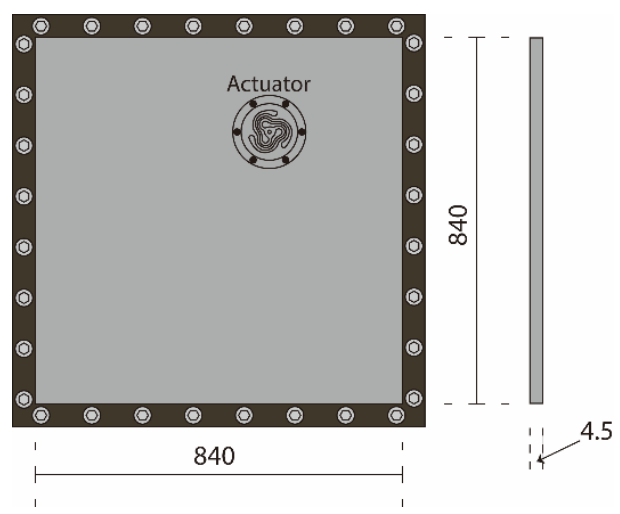

Fig. 4. Steel plate model.

Experimental identification of the steel plate was performed to obtain the natural frequencies, modal damping ratios and complex modal shape vectors for the 81 grid mesh points. Acceleration responses at all 81 grid points were measured under impact loading by a hammer and transfer function for all the grid points were experimentally identified with a software Me'scope. The frequency range of recording is between $0 \mathrm{~Hz}$ and $200 \mathrm{~Hz}$. The identified five modes of the plate are shown in Fig. 6 .

Based on the result of experimental identification, the total number of modes to be considered is selected to be 5 , implying that $N=5$. The 1 st $\sim 3 \mathrm{rd}$ modes were chosen as the modelled modes, i.e. $M=3$, and the 4 th $\sim 5$ th modes were considered as the residual modes, for which the spillover effect are minimized. The total number of sensors used for the modal filtering of the first three modes is 4 , implying that $S=4$.

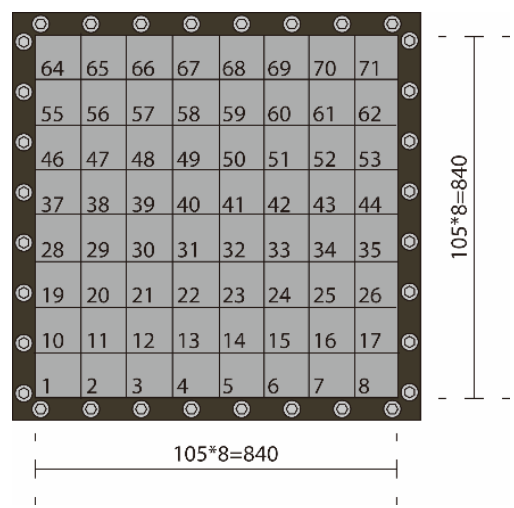

Fig. 5. 81 Point mesh on the steel plate.

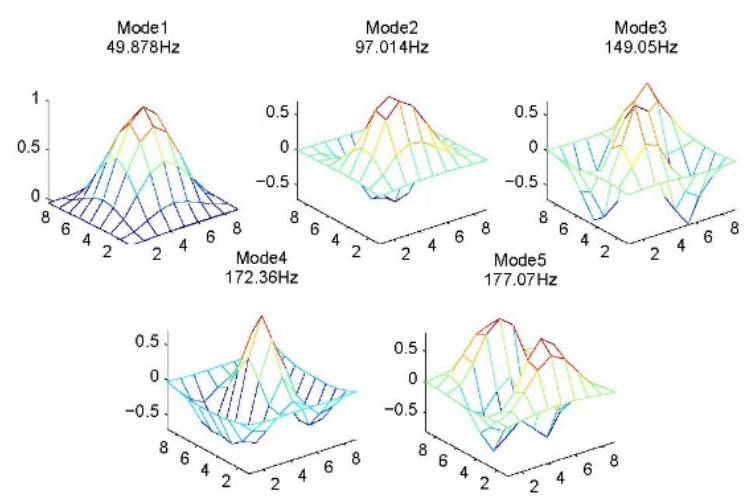

Fig. 6. Identified mode shapes.

\subsection{Optimal sensor placement}

The result of each step in the procedure described in Sec. 3 is shown here. The modal matrix $\boldsymbol{\Psi}$ evaluated at the $L$ grid points, such that $\boldsymbol{\Psi}^{\boldsymbol{T}} \boldsymbol{\Psi}=\boldsymbol{I}$ is obtained by modal identification of the plate. The number of the total points are chosen so that the number of pre-defined $P=41$ points are left after applying EFI to the lowest three modes. The remaining $P$ points are shown as blue circles in Fig. 7. It can be seen that the contribution of the points near the clamped edges to the linear independence of the target first three modes is less significant and effects for the state estimation are comparatively minor.

Next, all the possible sensor placement of 4 sensors within 41 point candidates are ranked in an increasing manner based on the value of $A_{s p i}$, as described in Sec. 2.1. The sensor placement for the smallest $\mathrm{A}_{s p i}=0.0705$ is at points $16,31,51,60$. 
However, since $A_{m a c}=\sum_{a=1}^{2} \sum_{b=a+1}^{3} \operatorname{MAC}\left(\Psi_{a}, \Psi_{b}\right)=0.6373$, which is significantly larger than the tolerance value $\mathrm{A}_{\text {maclim }}=0.1$, this sensor placement is not the optimal one.

By checking the next smallest sensor placement and comparing the value of $A_{\text {mac }}$ with the tolerance value $A_{\text {maclim }}$ until $A_{\text {mac }} \leq A_{\text {maclim }}$ is satisfied, the final optimal sensor placement is found to be at points $25,31,50$ and 61 with $A_{\text {spi }}=0.0948$ and $A_{m a c}=0.056$. The locations of the 4 sensors of the optimal placement are shown in Fig. 8.

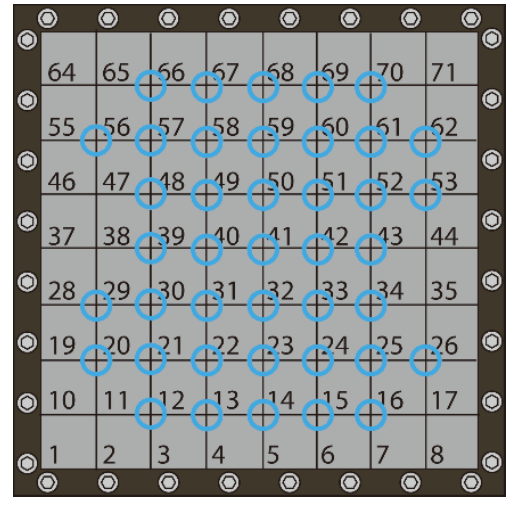

Fig. 7. Sensor candidates after EFI.

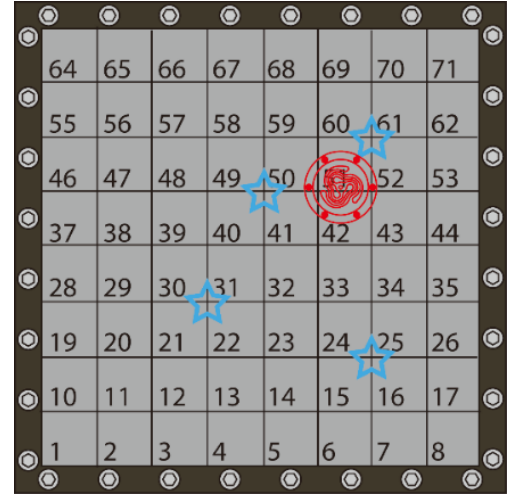

Fig. 8. Optimal sensor and actuator placement.

\subsection{Actuator placement}

EFI is used to remove the candidate actuator points which contain least information to the control modes to obtain 10 candidate actuator points (a pre-defined number) out of 81 grid points. Since only one actuator is used in this test, the spillover effects of the actuator can be checked through the modal participation of the residual modes on these 10 points using mode shape vectors. A point located between 51 and 52 is found to have a large participation on the control modes and small participation on the residual modes. The actuator is determined to be located at this point near the node of the 4th mode. The optimal placement of the actuator is shown in Fig. 8.

\subsection{Performance of modal filtering}

In order to evaluate the performance of the modal filtering with the optimal sensor placement, three cases are considered. Case A: the optimal sensor placement with $A_{s p i}=0.0948$ and $A_{m a c}=0.056$. Case B: a large spillover and small consistency sensor placement, such that $A_{s p i}=30.2283$ and $A_{m a c}=0.0776$. Case C: another large consistency and small spillover sensor placement, such that $A_{s p i}=0.0965$ and $A_{m a c}=0.7461$. The layout of the sensor placement for Case B and Case C are shown in Figs. 9 and 10, respectively.

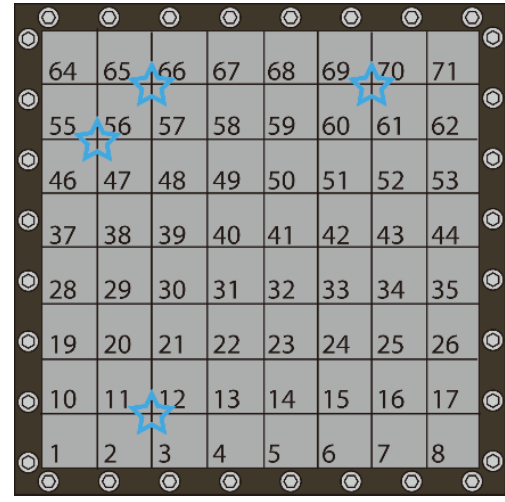

Fig. 9. Case B sensor placement

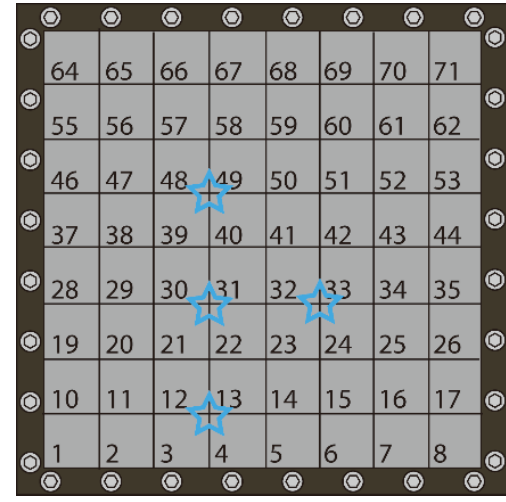

Fig. 10. Case $\mathrm{C}$ sensor placement.

The Fourier spectra of the modal filtering output using Eqn. (3) for the three cases are shown in Figs. 11 through 13 with the sampling ratio of $1024 \mathrm{~Hz}$. The natural frequency of each mode is indicated with a red arrow symbol. It can be observed in Case A that all the first three modes are filtered out and the spillover of the 4th and 5th are small, implying that all of the first three 
modes are observed and the modal filtering for optimal sensor placement enhances the target natural mode response while the other two residual modes are effectively reduced. Note that the spillover effect still exists here and refinement of the mesh (large $L$ ) is needed to achieve a reduced spillover effect.

In Case B, the performance of the modal filtering is excellent for the 2nd and 3rd mode. However, for the 1st mode not only the spillover effect is far greater than Case A but also the separation performance for the first modes is poor. The former is the result of the high value of $A_{s p i}$ while the latter is caused by the poor observation quality of the 1st mode, see Figs. 6 and 9 . All the four sensors are located near the nodes of the 1st mode.

In Case $\mathrm{C}$, although the spillover effect is small as in Case $\mathrm{A}$, the separation performance of modal filtering is very poor, especially for the 1st and 3rd modes. This result is explained as a natural consequence of the relatively large value of $A_{m a c}$.

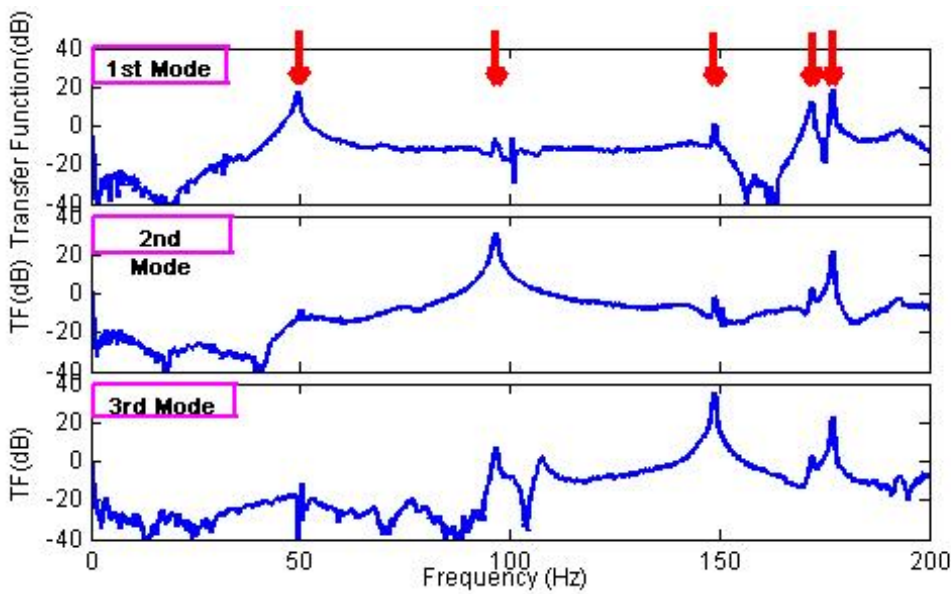

Fig. 11. Transfer function for MF output (Case A).

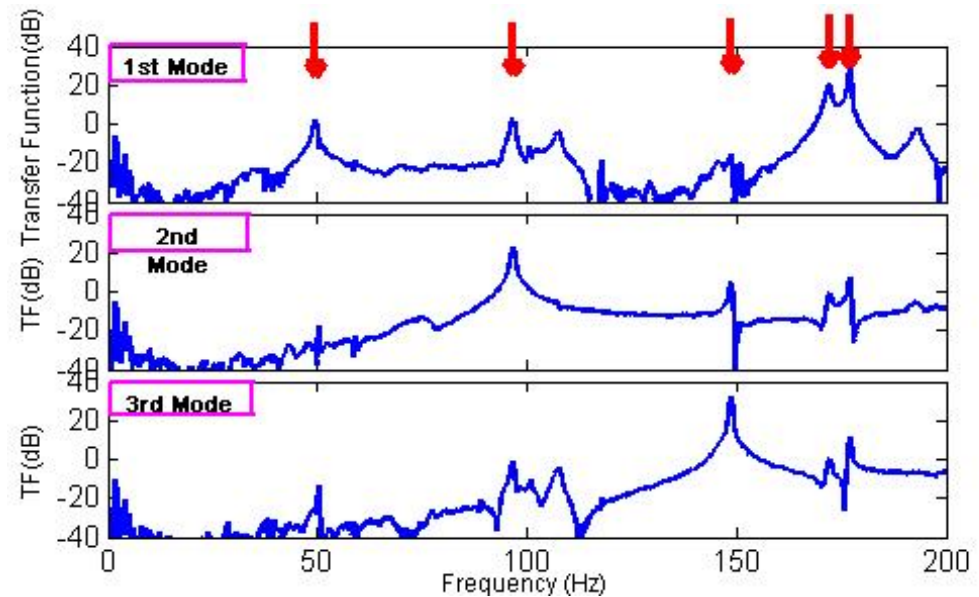

Fig. 12. Transfer function for MF output (Case B). 


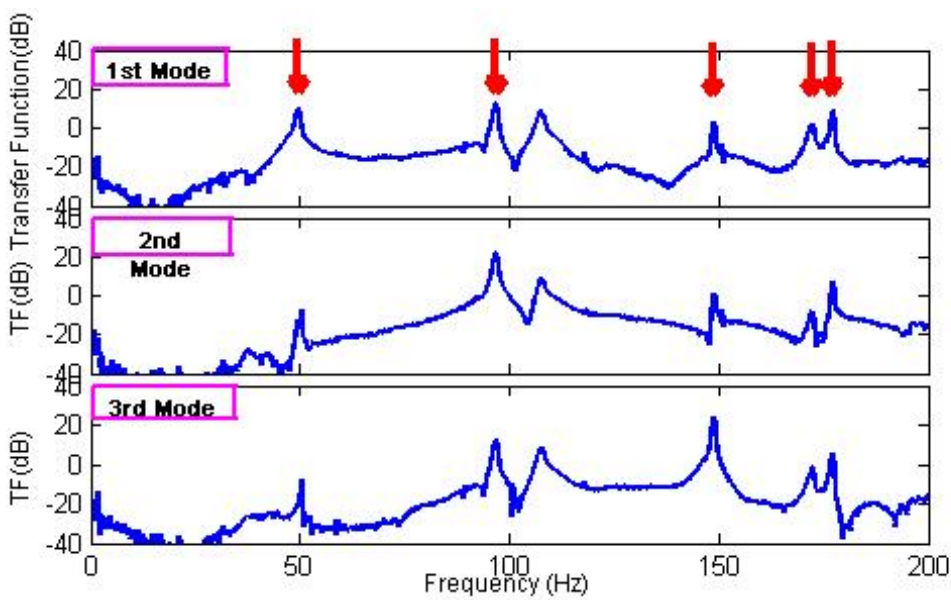

Fig. 13. Transfer function for MF output (Case C).

Figs. 14 to 16 show the filtered time domain performance of the lowest three modes for the three cases ranged from $0 \mathrm{~Hz}$ to $200 \mathrm{~Hz}$. It can be observed that only Case A can filter the three mode responses successfully, for Case B, since high spillover effect of the 4th and 5th modes involved in the first mode, it is difficult to observe the first mode responses from Fig. 14, however, good performance can be found in Figs. 15 and 16 that the second and third mode responses are filtered out successfully. Thus, a sensor placement with high value of $A_{s p i}$ does not mean all of the modes cannot be filtered in this method.

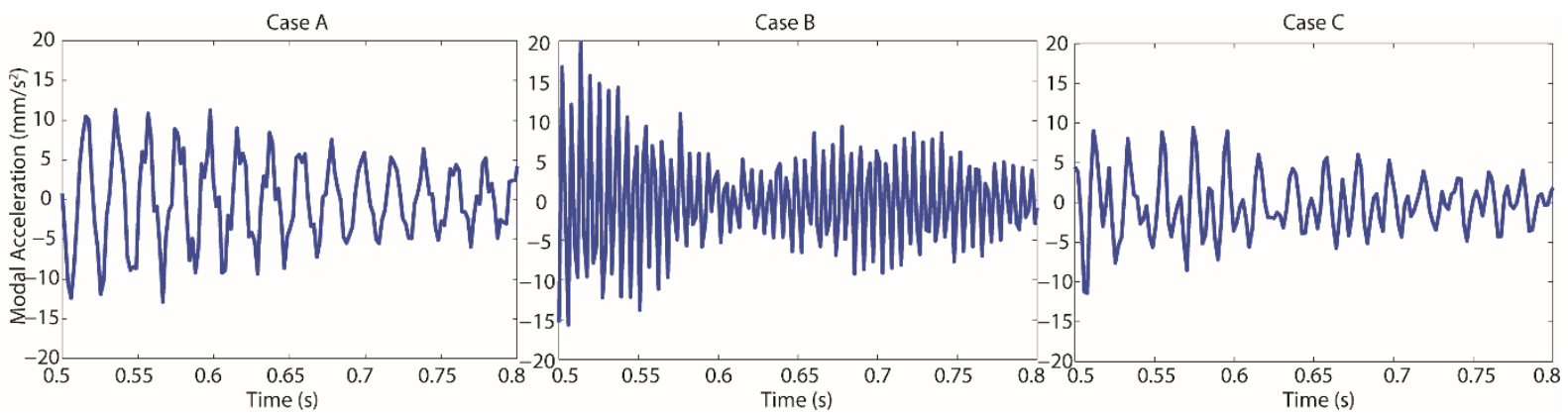

Fig. 14. First mode modal acceleration.

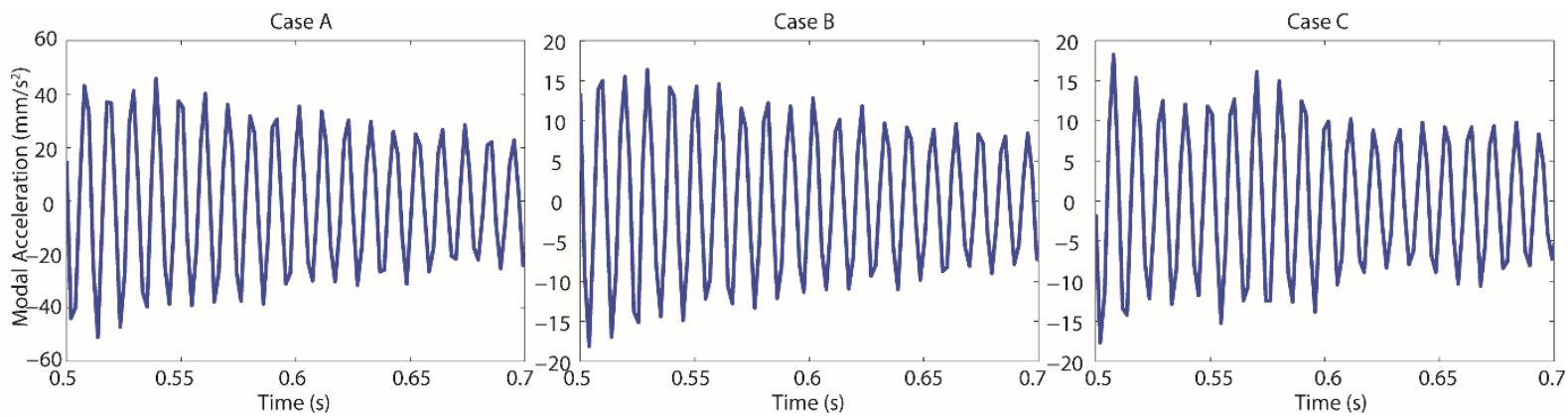

Fig. 15. Second mode modal accereration.
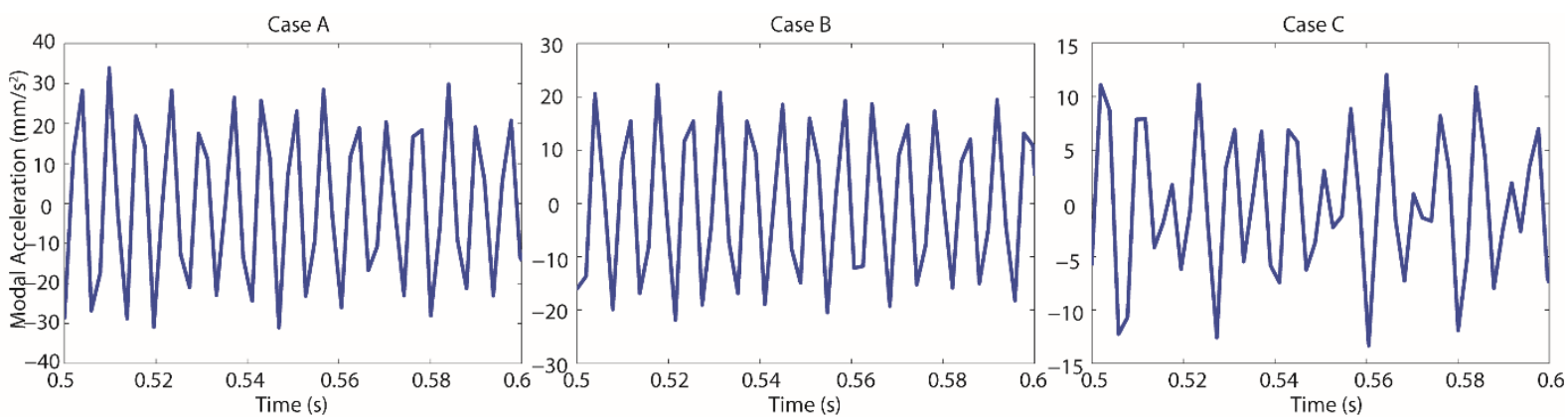

Fig. 16. Third mode modal accereration 


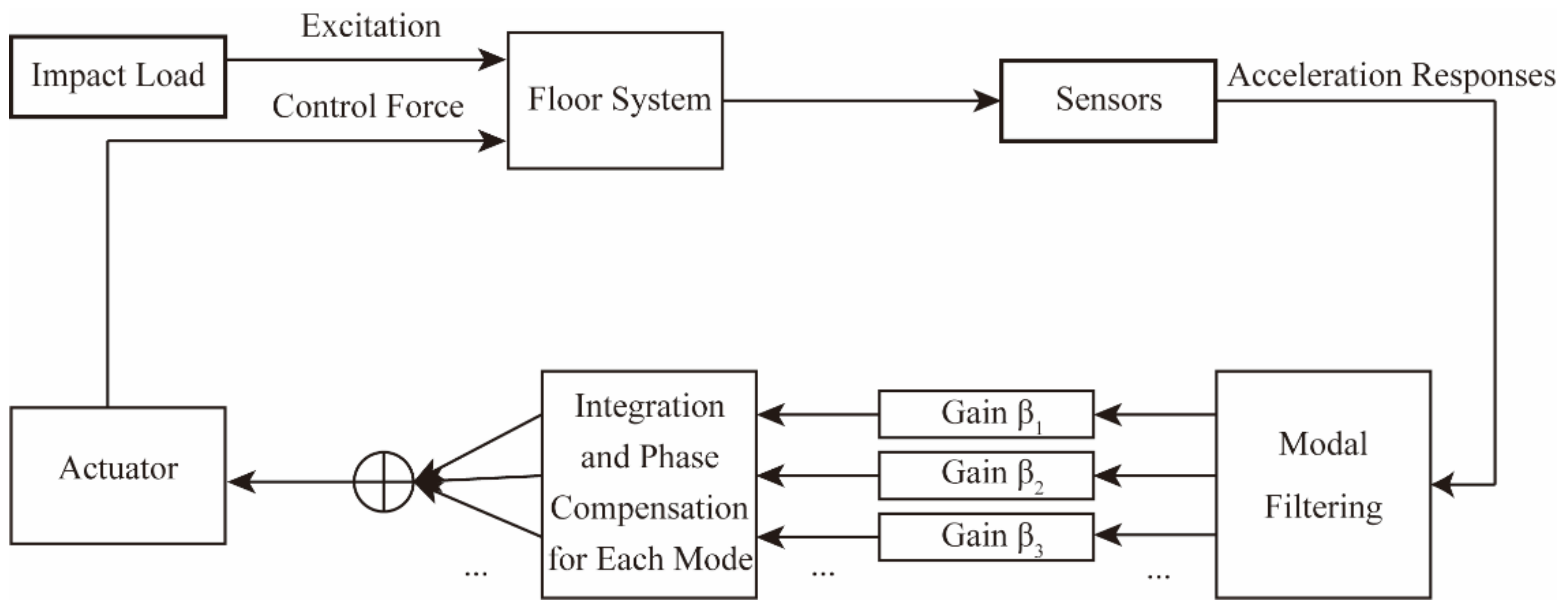

Fig. 17. Active control system.

\subsection{Active control}

Phase-compensated MDVF control shown in Sec.4 is combined with the proposed MF strategy for the reduction of all the control modes of the transient vibration of the steel plate under impact loading. The schematics of the control strategy is shown in Fig. 17. The steel plate shown in Fig.3 is used for the control performance verification test, in which four acceleration sensors and an actuator for the active control are installed on the steel plate. The electromagnetic actuator used in the active control is shown is Fig. 18. Weight and stroke of the inertial mass of the actuator is $1.8 \mathrm{Kg}$ and $\pm 8 \mathrm{~mm}$, respectively. Identification of the dynamic characteristics of the actuator was done using the MATLAB identification tool box. The identified dynamic characteristics of the actuator which relates the released inertial force with the input voltage is shown in Fig. 19. The natural frequency of the actuator is estimated as $21 \mathrm{~Hz}$.

The impact load to the plate is applied by a hammer which is shown in Fig. 3. The length of the hammer is $37 \mathrm{~cm}$ and the head diameter is $5.1 \mathrm{~cm}$. For all of the control tests, the hammer is knocked at point 21 with the approximately same strength, as shown in Fig. 20. The piezoelectric accelerometers and charge amplifiers are used for measurement of the steel plate vibration. A controller is synthesized based on the measured signal to reduce the vibrations associated with the modes that need to be controlled by increasing their corresponding modal damping. Gains of the control modes are incrementally increased in the range such that the system is stable.

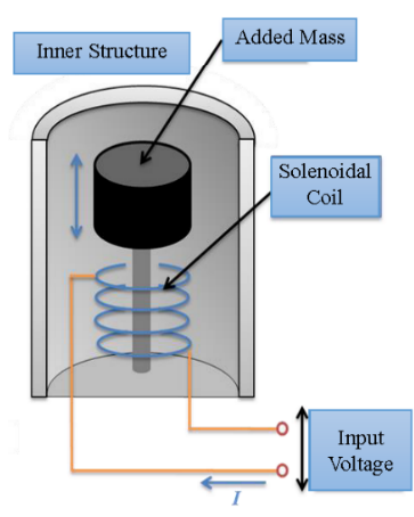

Fig. 18. Actuator for active control

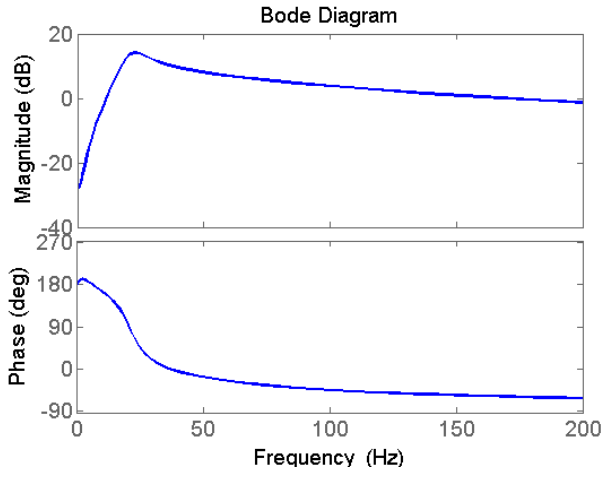

Fig. 19. Frequency response of actuator 


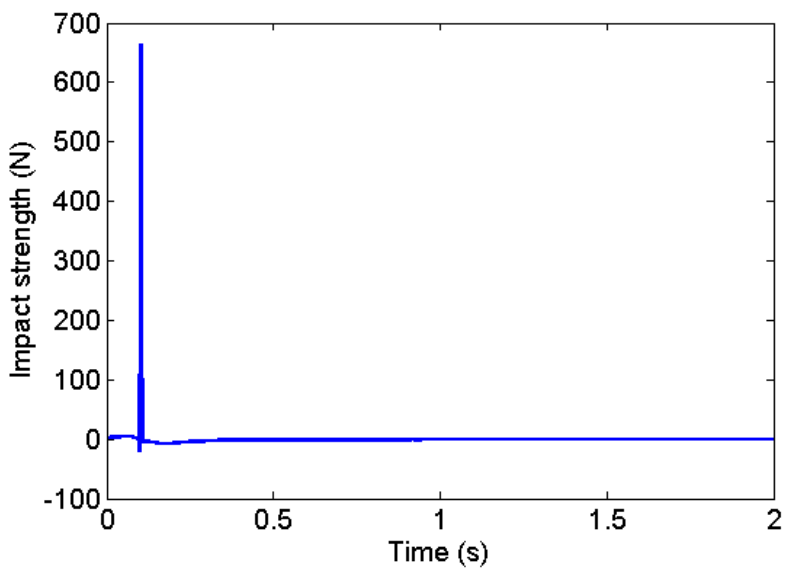

Fig. 20. Impact load excited by hammer.

Using the results of the modal filtering, the active control verification tests are performed using the optimal sensor placement. First of all, single mode control tests are conducted for the verification of the proposed sensor placement method. The results are shown in Figs.21, 23 and 25, in which the transfer function between the excitation force and the acceleration response at point 31 are compared for the cases with and without active control. The corresponding input voltages to the actuator from the DSP (digital signal processor) are shown in Figs. 22, 24 and 26. It can be seen that an effective modal filtering of desired modes were achieved from these figures. Also, the spillover effect of the $4^{\text {th }}$ and $5^{\text {th }}$ modes with small amplitudes can be observed from these figures.

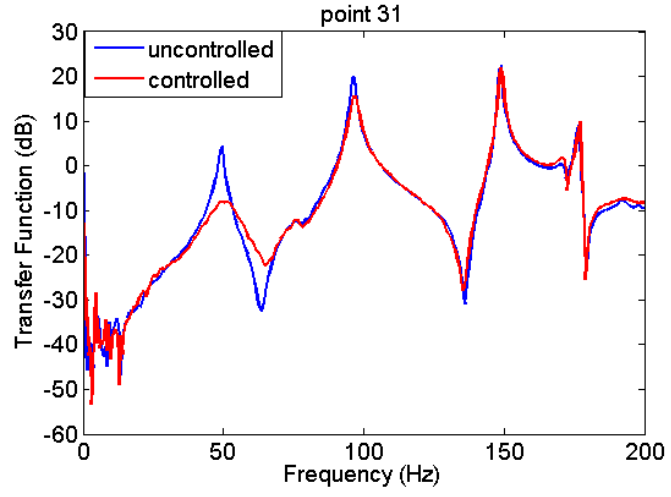

Fig. 21. Test result: first mode control at point 31 .

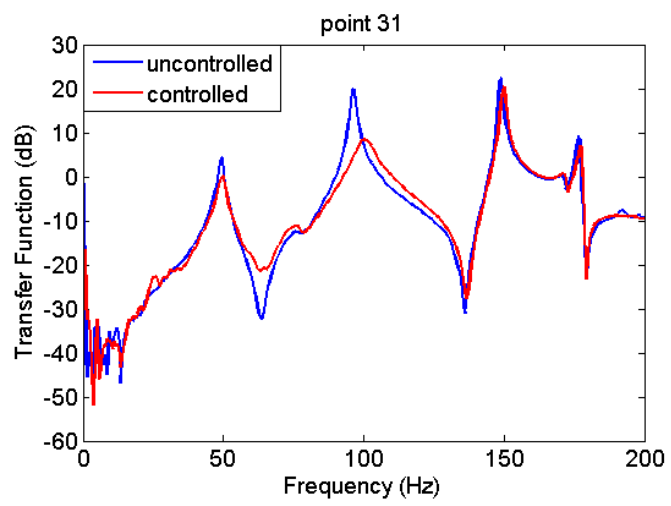

Fig. 23. Test result: second mode control at point 31.

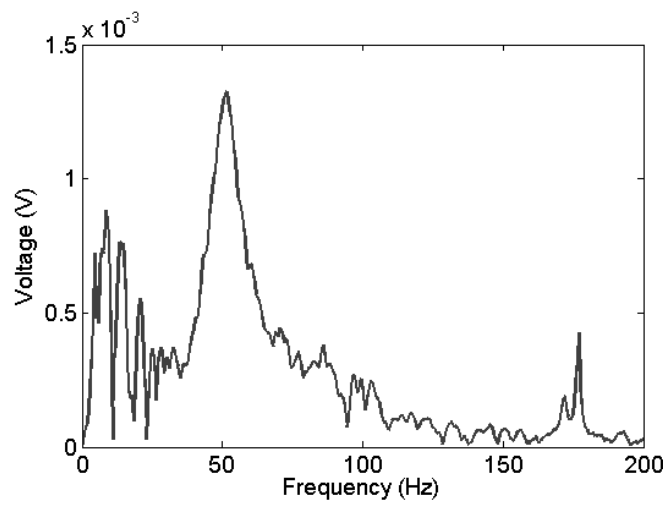

Fig. 22. Test result: actuator voltage for first mode control.

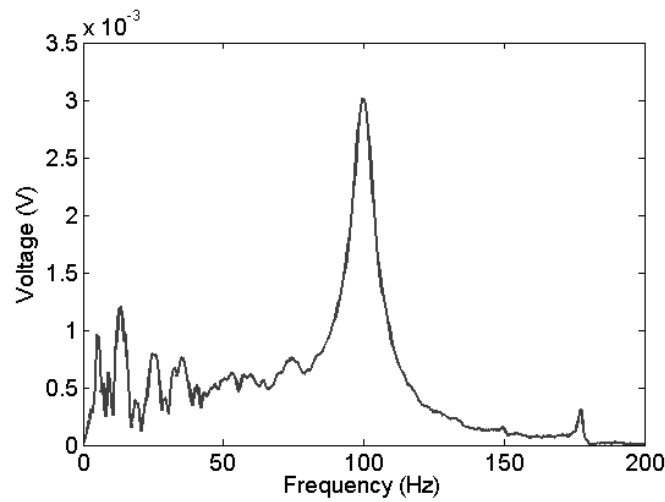

Fig. 24. Test result: actuator voltage for second mode control. 


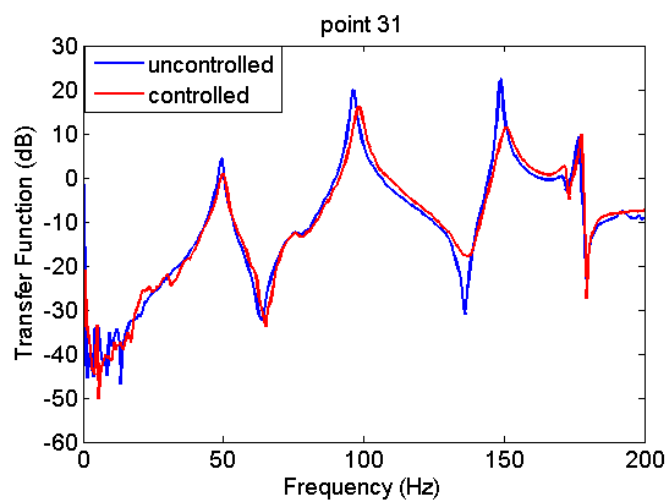

Fig. 25. Test result: third mode control at point 31.

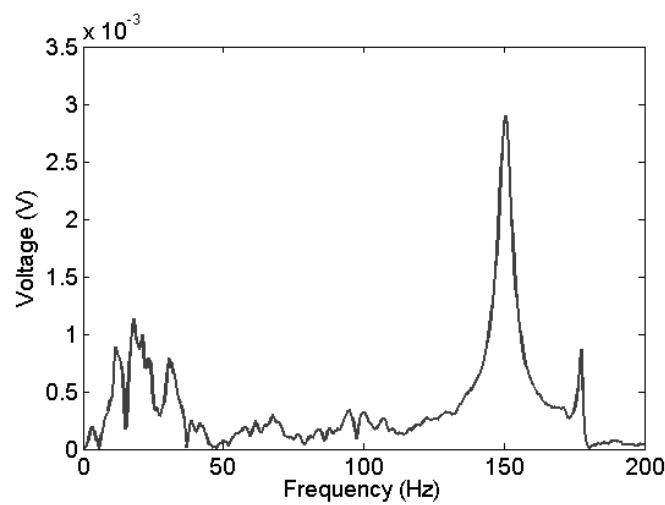

Fig. 26. Test result: actuator voltage for third mode control.

Control result of the lowest two modes and the corresponding input voltage to the actuator are also shown in Figs.27 and 28 , respectively. It is observed that the DSP offers a signal with little information of the third mode as well as small amplitude of the $4^{\text {th }}$ and $5^{\text {th }}$ modes to the actuator.

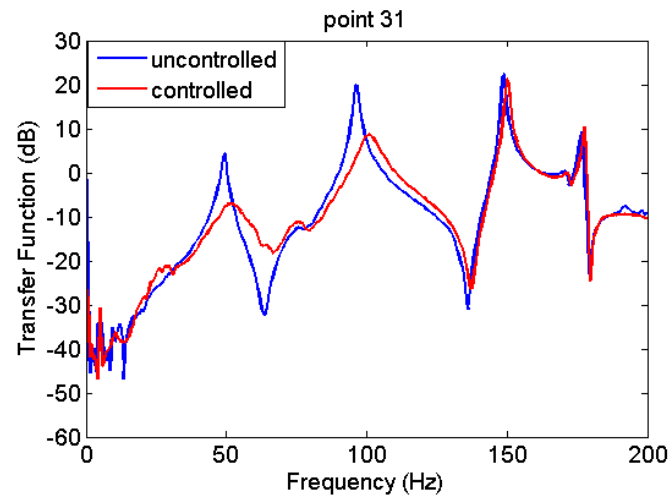

Fig. 27. Test result: lowest two modes control at point 31.

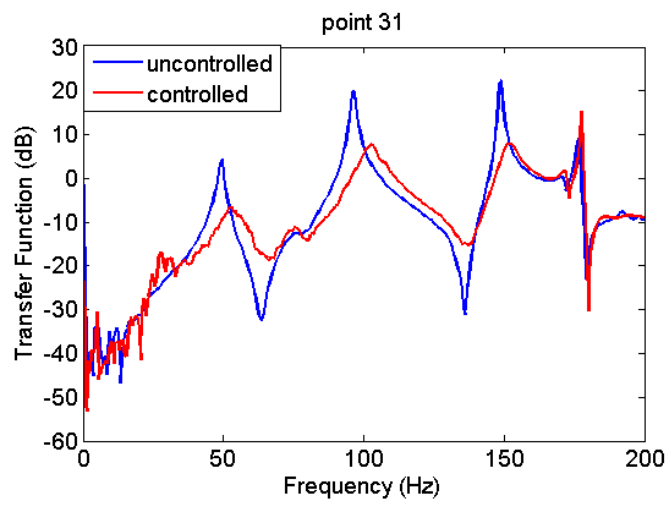

Fig. 29. Test result: Frequency domain response for the lowest three modes control at point 31 .

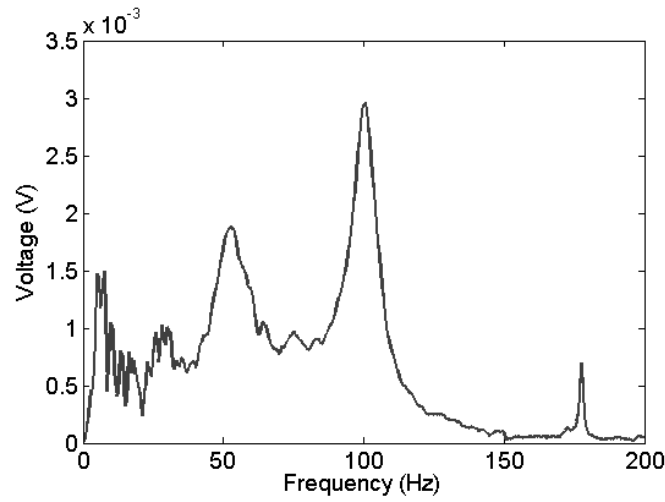

Fig. 28. Test result: actuator voltage for lowest two modes control.

Finally, the case of controlling of the lowest three modes is done. A high pass filter with a cut-off frequency of $20 \mathrm{~Hz}$ is used to avoid the low frequency noise. Figs. 29 and 30 show the frequency and time domain response for the cases with and without active control of point 31. Significant reduction of responses in the lowest three modes shown in the figures indicates that the independent modal control enables the system to increase the modal damping of each controlled mode to effectively reduce the vibration of the steel plate. Since the actuator is placed in the vicinity of the node of the 4th mode shape, the change of the peak response of this mode is not significant. On the other hand, the increase of the peak of the 5 th mode response is a strong indication 
of the spillover effect. The superior control performance for the lowest three modes can be explained by effective reduction of the spillover effects for the lowest three modes achieved by the optimal sensor placement, with the use of the minimum spillover method and EFI.

\section{Conclusions}

In order to develop effective active control technique for reducing human-induced vibration and noise of building floors, a procedure for the optimal sensor placement applicable to active control of plate vibration is proposed. The aim of the proposed procedure is to provide a solution to the problems of ensuring the state estimate ability, minimization of the spillover effect, consideration for orthogonality of the natural vibration modes and system observability. Effectiveness of the proposed procedure and achieved control performance are examined by a series of verification tests using a rectangular steel plate and an active control system.

The procedure for the proposed sensor placement approach is effective in the reduction of the spillover effect of the residual modes and enhancing the separation performance of the modeled modes. First, the effective independence method removes the sensor location candidates with small contribution to the state estimate of the controlled modes, and then in the second step, the minimum spillover method is used to rank all the possible sensor placements in the order of severer spillover effects, and finally the sensor placement with the minimal spillover effects and MAC value is chosen as the optimal one.

The performance of the modal filtering with the sensor placement determined by the proposed procedure is compared with other two cases. Effectiveness of the sensor placement by the proposed method is successfully verified by comparison. Both of the orthogonality and spillover effect are very important for the abstraction of desired modes. For the case of high spillover indicator $A_{s p i}$, only the first mode cannot be filtered, thus a sensor placement with high value of spillover indicator $A_{s p i}$ does not mean all of the modes cannot be filtered in this method.

Control tests also showed the effective of the modal filtering using the proposed sensor placement by conducting the individual mode control. Multi-modes control were successfully achieved using the optimal sensor placement. By reducing the spillover effect and enhancing the orthogonality property, significant reduction of the response was achieved in the controlled modes.

\section{Acknowledgements}

The authors wish to thank the Japanese Government (Monbukagakusho: MEXT) for providing the scholarship for the first author to conduct the study in this paper.

\section{References}

[1] Iván M. Díaz and Paul Reynolds, 'On-off Nonlinear Active Control of Floor Vibrations', Mechanical Systems and Signal Processing, 24 (2010), 1711-26.

[2] Hugo Bachmann, 'Case Studies of Structures with Man-Induced Vibrations', Journal of Structural Engineering, 118 (1992), 631-47.

[3] Linda M. Hanagan, and Thomas M. Murray, 'Active Control Approach For Reducing Floor Vibration', Journal of Structural Engineering, 123 (1997), 1497-505.

[4] Linda M. Hanagan, Thomas M. Murray and Kamal Premaratne, 'Controlling Floor Vibration with Active and Passive Devices', The Shock and Vibration Digest, 35 (2003), 347-65.

[5] Malcolm J. Hudson, and Paul Reynolds, 'Implementation considerations for active vibration control in the design of floor structures', Engineering Structures, 44 (2012): 334-58.

[6] Mark J. Balas, 'Direct Velocity Feedback Control of Large Space Structures', Journal of Guidance, Control, and Dynamics, 2 (1979), 252-53.

[7] Iván M. Díaz and Paul Reynolds, 'Acceleration Feedback Control of Human-Induced Floor Vibrations', Engineering Structures, 32 (2010), 163-73.

[8] Iván M. Díaz, and Paul Reynolds, 'Robust saturated control of human-induced floor vibration via a proof-mass actuator', Smart Materials and Structures, 18 (2009), 125024. 
[9] Donald Nyawako, Paul Reynolds and Emma Hudson, 'Incorporating a disturbance observer with direct velocity feedback for control of human-induced vibrations', SPIE Smart Structures and Materials + Nondestructive Evaluation and Health Monitoring. International Society for Optics and Photonics, 2016.

[10] Malcolm J. Hudson, Paul Reynolds and Donald Nyawako, 'Efficient design of floor structures using active vibration control', ASCE Structures Congress, Vol.401, 2011.

[11] Emiliano Pereira, Iván M. Díaz, Emma J. Hudson and Paul Reynolds, 'Optimal control-based methodology for active vibration control of pedestrian structures', Engineering Structures, 80 (2014): 153-62.

[12] L. Meirovitch and H. Baruh, 'The Implementation of Modal Filters for Control of Structures', Journal of Guidance, Control, and Dynamics, 8 (1985), 707-16.

[13] Q. Zhang, R. J. Allemang and D. L. Brown, 'Modal Filter: Concept and Applications', Proceedings of International Modal Analysis Conference, 1990, 487-96.

[14] S. Shelley, L. Freudinger, R. J. Allemang and Q. Zhang, 'Implementation of a Modal Filter on a Five Meter Truss Structure', Proceedings of the International Modal Analysis Conference - IMAC, 1991, 1036-44.

[15] L. Meirovitch and H. Baruh, 'On the Problem of Observation Spillover in Self-Adjoint Distributed-Parameter Systems', Journal of Optimization Theory and Applications, 39 (1983), 269-91.

[16] Mark J. Balas, 'Active Control of Flexible Systems', Journal of Optimization Theory and Applications, 25 (1978), 415-36.

[17] Mark J. Balas, 'Feedback Control of Flexible Systems', IEEE Transactions on Automatic Control, 23 (1978), 673-79.

[18] S. Cinquemani, D. Ferrari and I. Bayati, 'Reduction of Spillover Effects on Independent Modal Space Control through Optimal Placement of Sensors and Actuators', Smart Materials and Structures, 24 (2015), 85006.

[19] Daniel C. Kammer, 'Sensor Placement for on-Orbit Modal Identification and Correlation of Large Space Structures', Journal of Guidance, Control, and Dynamics, 14 (1991), 251-59.

[20] Nariyuki Kawabata and Hisao Fukunaga, 'Vibration Measurement/Control of CFRP Laminated Plates Based on the Optimal Placement of Sensors/Actuators Part I: Numerical Simulation(In Japanese)', Journal Of The Japan Society For Aeronautical And Space Science, 52 (2004), 499-506.

[21] Nariyuki Kawabata and Hisao Fukunaga, 'Vibration Measurement/Control of CFRP Laminated Plates Based on the Optimal Placement of Sensors/Actuators Part 2: Experiment(In Japanese)', Journal Of The Japan Society For Aeronautical And Space Science, 53 (2005), 295-301.

[22] Dennis R. Morgan, 'An Adaptive Modal-Based Active Control System', The Journal of the Acoustical Society of America, 89 (1991), 248-56.

[23] Dongchang Sun and Liyong Tong, 'Vibration Control of Plates Using Discretely Distributed Piezoelectric Quasi-Modal Actuators/sensors', AIAA Journal, 39 (2001), 1766-72.

[24] Tomas Hanis and Martin Hromcik, 'Optimal Sensors Placement and Spillover Suppression', Mechanical Systems and Signal Processing, 28 (2012), 367-78.

[25] D. S. Li, H. N. Li and C. P. Fritzen, 'The Connection between Effective Independence and Modal Kinetic Energy Methods for Sensor Placement', Journal of Sound and Vibration, 305 (2007), 945-55.

[26] Daniel C. Kammer and Michael L. Tinker, 'Optimal Placement of Triaxial Accelerometers for Modal Vibration Tests', Mechanical Systems and Signal Processing, 18 (2004), 29-41.

[27] Daniel C. Kammer, 'Sensor Set Expansion for Modal Vibration Testing', Mechanical Systems and Signal Processing, 19 (2005), 700-713.

[28] Randall J. Allemang, 'The Modal Assurance Criterion-twenty Years of Use and Abuse', Sound and Vibration, 37 (2003), 14-23. 